\title{
RADICCHIO (Cichorium intybus) PARA EXPORTACIÓN: EVALUACIÓN DE VARIEDADES, TAMAÑOS DE PLÁNTULAS PARA TRANSPLANTE Y COBERTURA PLÁSTICA SOBRE EL SUELO ${ }^{1}$
}

\author{
Martín Solís-Salazar², María Isabel González-Lutz², Dennis Mora-Acedo³, Alfredo Durán-Quirós ${ }^{3}$
}

\begin{abstract}
RESUMEN
Radicchio (Cichorium intybus) para exportación: evaluación de variedades, tamaños de plántulas para transplante y cobertura plástica sobre el suelo. Se evaluaron dos variedades de radicchio (Leonardos y Verona), tres tamaños de plántula para trasplante y cobertura del suelo con plástico, sobre la calidad para exportación. El experimento se realizó en Cartago, Costa Rica en los meses julio a setiembre del 2005, durante la estación lluviosa. La variedad Leonardos produjo una mayor exportabilidad. El tamaño de las plántulas del trasplante no tuvo influencia sobre la exportabilidad al igual que el uso de cobertura de plástico.
\end{abstract}

Palabras claves: Cichorium intybus, endivias, chicoria, criterio de cosecha, pérdidas de producción.

\begin{abstract}
Radicchio (Cichorium intybus) for export: variety evaluation, size of seedlings for transplant and the use of a soil plastic cover. Effect of variety (Leonardos and Verona), size of plant for transplant (small, the usual, large) and soil condition (cover and non-covered with plastic) on exportability were evaluated. The criteria of exportability was compared between the harvester and the packer. The experiment was made in Cartago, Costa Rica, in the months July to September of 2005, during the rainy season. Variety Leonardos showed the highest exportability: a historical $30 \%$ can be raised to $75 \%$. Size of plants for transplant and covering with plastic had no effect.
\end{abstract}

Key words: Cichorium intybus, endives, chicory, harvesting criterion, production loss.

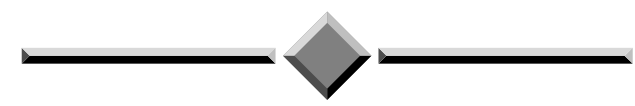

\section{INTRODUCCIÓN}

El radicchio (Cichorium intybus) es una hortaliza del tipo de las endivias que se produce en Costa Rica desde hace tres años para la exportación por vía marítima. Es una hortaliza de hoja, de gran importancia comercial, cuyo principal productor a nivel mundial es Italia (Pimpini et al. 2002). En Estados Unidos el radicchio es producido en California y Florida, mientras que en el mercado internacional destacan Guatemala, Italia, Chile y otros países de América Central como los principales proveedores de radicchio (Solutions Associates 2006).

En la mayoría de los países donde se consume el radicchio, se utiliza fundamentalmente como un componente de ensaladas frescas, o bien se expende directamente.

Recibido: 28 de setiembre, 2006. Aceptado: 26 de agosto, 2008. Proyecto de investigación.

2 Escuela de Estadística, Facultad Ciencias Económicas, Universidad de Costa Rica. martin12cr@yahoo.com; mariaisabel.gonzalezlutz@ucr.ac.cr

3 Estación Experimental Fabio Baudrit, Facultad de Ciencias Agroalimentarias, Universidad de Costa Rica. jose.duran@ucr.ac.cr; dennis. mora@ucr.ac.cr. 
La producción de plantas de radicchio se puede realizar mediante dos métodos, siembra directa o a través de almácigo-trasplante. El empleo de plantines de radicchio para transplantar, presenta ventajas que se destacan sobre el método de siembra directa, éstas son principalmente: menor costo de semillas, optimización de la germinación, menor empleo de mano de obra, posibilidades de anticipar la entrada al mercado en casi un mes, mejor control de malezas y mayor calidad del producto final (Ramponi 2000).

En relación a las temperaturas requeridas por el cultivo de radicchio, Pimpini et al. (2002) afirman que las temperaturas óptimas de crecimiento se encuentran entre 15 y $20^{\circ} \mathrm{C}$, y que la temperatura base de crecimiento está en torno a los 8 a $10^{\circ} \mathrm{C}$.

Al inicio del desarrollo, las hojas se presentan extendidas y verdes, las cuales a partir de un determinado número de éstas sumado a temperaturas ambiente bajas, comienzan a adquirir una tonalidad rojiza a morada, además de cambiar su disposición hacia una forma más compacta, en que las hojas más viejas envuelven a las más nuevas para que éstas últimas no se extiendan (Pimpini et al. 2002). De esta forma el radicchio forma una cabeza similar al repollo, pero de color morado con las venas blancas. Algunas variedades tienden a tener forma esférica, mientras que otras son alargadas.

Durante las primeras experiencias de producción en la zona de Cartago, Costa Rica, el porcentaje de cabezas exportadas tuvo un promedio de tan solo el 30 $\%$, por la desuniformidad de tamaño que presentaron las plantas en la época en que debían ser cosechadas. Esto causa que la producción de este cultivo no sea rentable, porque la exportación es por vía marítima, y se requiere cosechar todo el producto en el término de una semana, se requiere sembrar al menos un $50 \%$ más de plantas, para cosechar en una semana las cantidades acordadas con el mercado. Este problema del cultivo del radicchio ya ha sido reportado por varios autores (Rangarajan e Ingall 2001, Carrasco et al. 1998, Lazzarin et al. 2000, Carvajal y Krarup 1997). Por otra parte, Reich et al. 2000, han informado que los bajos porcentajes de formación de cabezas es un problema general de la mayoría de variedades de radicchio.
Estudios realizados por Suhonen (1991) y por Carvajal y Krarup (1997) indican que las distintas variedades del radicchio pueden comportarse diferente entre sí en la producción y muestran que el porcentaje de cabezas que pueden ser vendidas es significativamente diferente entre algunas variedades.

Otro de los factores que se cita como responsable de la variabilidad al momento de cosecha de las plantas, es la desuniformidad de las plantas de almácigo al momento del transplante. Al respecto Lazzarin et al. (2000), cita que el aumento de la uniformidad de las plantas de transplante mediante el uso de micro plantas, provocó una mejoría en la proporción de plantas que alcanzan el punto de corte a una edad determinada.

Otra posible causa del bajo rendimiento de la cosecha para exportación, es que el criterio de exportabilidad del producto está basado en consideraciones de tipo cualitativo y por lo difíciles que son de definir, pueden causar discrepancias entre los responsables de la cosecha y los de la selección, puesto que un cosechador selecciona al tacto lo que considera exportable, pero luego es el empacador quien finalmente decide, también usando el tacto, lo que es adecuado para la exportación. Un mal criterio de selección puede ocasionar que radicchios exportables no sean cosechados o sean rechazados al momento del empaque, o bien que radicchios que no tienen condiciones, se exporten, disminuyendo así la calidad del producto.Por otra parte, estudios previos (Rangarajan e Ingall 2001, Reich et al. 2000), han demostrado que la cobertura de suelo con un plástico negro-plata aumenta la proporción de cabezas cosechadas, así como su peso individual y adelanta la cosecha. Además, Reich y Coffey (2001), encontraron que todos los colores de plásticos evaluados (negro, blanco o negro-plata), casi duplicaron la producción, con respecto a la ausencia de cobertura.

Los objetivos de este experimento fueron evaluar el efecto de dos variedades, tres tamaños de plántulas de trasplante y el uso de una cobertura plástica del suelo, sobre la exportabilidad del radicchio, así como evaluar la influencia de la aplicación de los criterios de exportabilidad del cosechador y del empacador del producto, sobre el rendimiento final de la producción. 


\section{MATERIALES Y MÉTODOS}

El experimento se realizó en Cartago, Costa Rica en los meses julio a setiembre de 2005 durante la estación lluviosa. En el Cuadro 1 se observan las principales características químicas del suelo donde se realizó el ensayo.

Con relación a las condiciones climáticas del experimento, según el Instituto Meteorológico Nacional ${ }^{4}$, en la Estación Meteorológica Linda Vista, en El Guarco de Cartago, los valores promedios mensuales para el periodo 1951-2004, indican que en la época en que se realizó el experimento, el más bajo brillo solar del año, con valores de 4,2 a 4,3 horas al día, con temperaturas medias cercanas a los $20^{\circ} \mathrm{C}$, mínimas alrededor de 14 ${ }^{\circ} \mathrm{C}$ y máximas alrededor de $25{ }^{\circ} \mathrm{C}$, humedad relativa alrededor del $85 \%$ y precipitaciones que variaron entre 126 y 253 litros por metro cuadrado.

La siembra se hizo en camas de 1,20 m de ancho, con tres hileras de plantas distanciadas por $30 \mathrm{~cm}$ entre hileras y una distancia entre plantas de $30 \mathrm{~cm}$. La distancia entre camas de siembra fue de $1 \mathrm{~m}$. El manejo del cultivo fue el mismo que se acostumbra en las siembras para la exportación.

A nivel de campo se evaluaron los siguientes tratamientos:

- Cobertura plástica (con y sin cobertura del suelo)

- Variedades: Leonardos y Verona.

- Desarrollo vegetativo del tamaño del almácigo: macroplantas, plantas medianas o testigo y microplantas.

Se usó cobertura de plástico de color plateado en la parte superior y negro en la cara que queda en contacto con el suelo.

Se usaron tres tamaños de plántulas para trasplante, las macroplantas (plántulas de mayor tamaño que lo acostumbrado) estuvieron 40 días en condición de almácigo en invernadero, tenían una altura a la punta de la hoja de 18 a 20 centímetros y seis hojas. Las plantas testigo o de tamaño mediano (plántulas del tamaño acostumbrado o normal que los productores utilizan para transplantar) estuvieron en invernadero por un período de crecimiento de 32 días, con una altura de 15 a $17 \mathrm{~cm}$ a la punta de la hoja y cinco hojas. Las microplantas (plántulas de menor tamaño a lo acostumbrado) por su parte, tuvieron un período de desarrollo en invernadero de 24 días, alcanzando 10 a $12 \mathrm{~cm}$. de altura a la punta de la hoja y cuatro hojas (Figura 1).

Independiente del tamaño de plántula, se uniformó entre el número de hojas y tamaño, además todas se transplantaron al campo el mismo día, para asegurar que se encontraran enfrentadas a las mismas condiciones. Todas fueron tratadas en el vivero bajo las mismas condiciones de manejo.

Se utilizó un diseño irrestricto aleatorio con un arreglo factorial de tratamientos $2 \times 2 \times 3$ (cobertura $\mathrm{x}$ variedad $\mathrm{x}$ tamaño al transplantar), que produjo 12 tratamientos, y cada uno fue repetido seis veces (seis

Cuadro 1. Características químicas del suelo de la zona de producción en donde se realizó el experimento de radicchio. Cartago, Costa Rica, 2005.

\begin{tabular}{|c|c|c|c|c|c|c|c|c|c|c|c|c|c|}
\hline pH & \multicolumn{4}{|c|}{$\operatorname{cmol}(+) / 1$} & \multirow[t]{2}{*}{ Acidez } & \multirow[t]{2}{*}{ CICE } & \multirow{2}{*}{$\begin{array}{c}\text { \% Satur. } \\
\text { Acidez }\end{array}$} & \multicolumn{6}{|c|}{$\mathrm{mg} / \mathrm{l}$} \\
\hline $\mathrm{H}_{2} \mathrm{O}$ & $\mathbf{C a}$ & Mg & $\mathbf{K}$ & $\mathbf{S}$ & & & & $\mathbf{P}$ & $\mathbf{C u}$ & $\mathrm{Fe}$ & Mn & $\mathbf{Z n}$ & B \\
\hline 4,99 & 16,6 & 2,78 & 0,98 & 14,2 & 0,24 & 20,6 & 1,17 & 39,3 & 10,8 & 432 & 13,8 & 5,1 & 1,28 \\
\hline
\end{tabular}

Solución Extractora: KCl-OLSEN.

\footnotetext{
Instituto Meteorológico Nacional, Gestión de Información y Comercialización. Promedios mensuales de datos climáticos. Estación Linda Vista - El Guarco - Cartago, Periodo 19512004.
} 


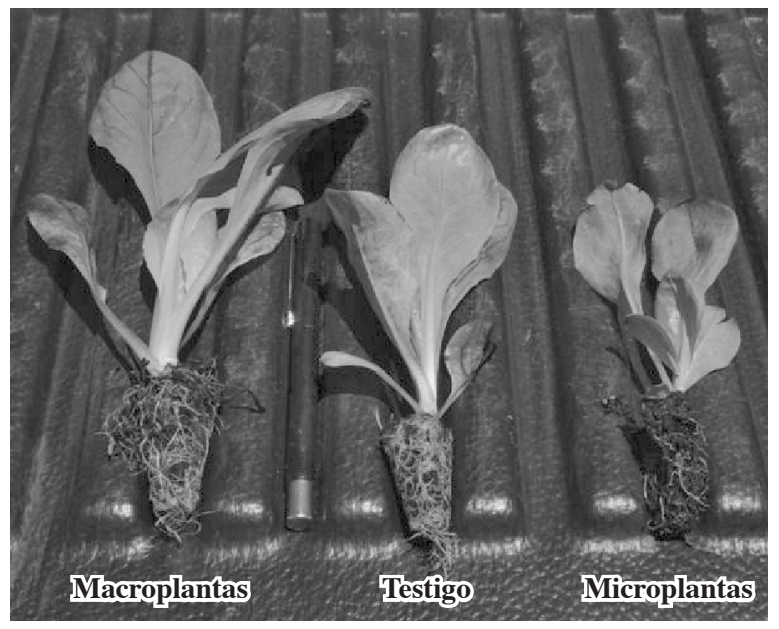

Figura 1. Tamaños de plántulas utilizadas en la evaluación de radicchio para exportación. Cartago, Costa Rica. 2005.

metros cuadrados de cultivo por tratamiento) para un total de 648 plantas experimentales.

Se cultivó en doce camas de siembra cada una con doce metros cuadrados de cultivo experimental. La unidad experimental fue un metro cuadrado de cultivo con tres líneas de tres plantas cada una. Entre una y otra unidad experimental se sembró un espacio con seis plantas de cultivo no experimental, distribuidas en dos líneas horizontales de tres plantas cada línea. Cada metro cuadrado tuvo la aplicación de un tratamiento asignado de manera aleatoria.

Tanto las plantas experimentales y las ubicadas en los bordes de las parcelas recibieron el paquete tecnológico usual para producciones destinadas a la exportación. La fertilización se realizó usando una medida para aplicar una dosis de fertilizante igual para todas las plantas del experimento.

Se realizaron dos cosechas de producto, en la primera fue recolectado todo lo que se consideró exportable, además de los radicchios que se consideraron pasados, en la segunda se cosechó el resto, incluyendo aquellos que aún les faltaba desarrollo y que por lo tanto sus hojas no se habían acomodado para formar la cabeza (no cabeceados). Los radicchios no cabeceados se cosecharon para cuantificarlos dentro de lo no exportable.
Los radicchios cosechados fueron clasificados por tres empacadores con experiencia en seleccionar y empacar para la exportación.

Se evaluó la exportabilidad de la producción, con base en los siguientes criterios:

- La firmeza del radicchio en su parte superior y a los lados, se clasificó como exportable cuando se consideró que la cabeza era firme al tacto y el corazón pequeño (cilindro central del radicchio).

- Circunferencia del radicchio en centímetros.

- Tamaño (alto) del corazón en centímetros, para lo cual se partieron los radicchios en dos mitades. La norma que se utiliza comúnmente es que la circunferencia debe ser como mínimo 6,28 veces el alto del corazón, en caso de no ser así el radicchio se considera no exportable por exceso de tamaño o "pasado".

- Color, medido con una escala visual de uno a cinco, donde uno es la presencia de color morado de más baja intensidad y cinco el de más alta intensidad (Figura 2).

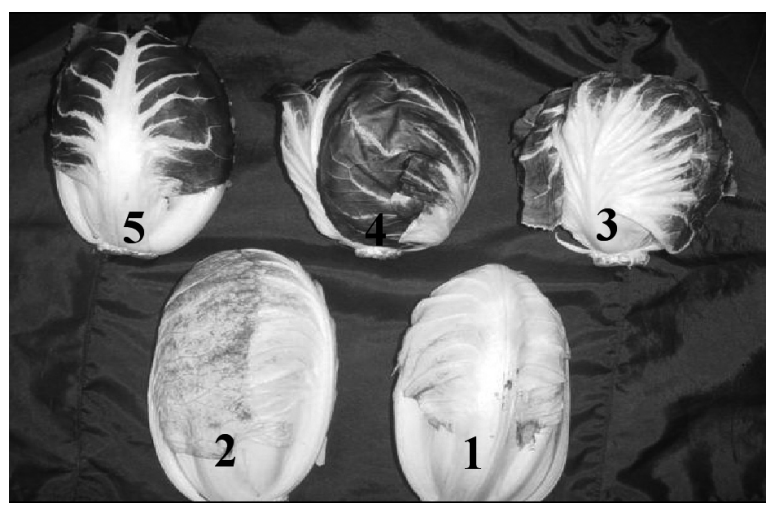

Figura 2. Escala de color utilizada para clasificar los radicchios. Cartago, Costa Rica. 2005.

Todos los análisis de resultados se efectuaron con base en el paquete estadístico STATA. Mediante el módulo Logístic, se realizaron análisis de regresión logística con corrección por multinivel, para evaluar la influencia de los factores del diseño (variedad, tamaño plántulas y cobertura plástica) y sus interacciones, 
sobre tres variables respuesta: exportabilidad total, exportabilidad si se cosechara en una semana determinada y radicchios perdidos (que murieron durante su desarrollo). La corrección por multinivel se utilizó para corregir el efecto de la correlación entre las mediciones en los radicchios de cada metro cuadrado de cultivo. Los coeficientes obtenidos de las regresiones se interpretaron con respecto a la variedad Verona, plantas testigo (de tamaño intermedio) y suelo sin cobertura. Mediante datos descriptivos, se exploró cuán uniforme es el criterio que el cosechador y el empacador tienen de la exportabilidad del producto.

\section{RESULTADOS Y DISCUSIÓN}

La primera cosecha se realizó a los 67 días del transplante y se colectó la mayor parte del cultivo (Cuadro 2). De los radicchios de la variedad Leonardos recolectados en esta primera cosecha, el $38,7 \%$ se declaró no adecuado para exportar porque el tamaño del corazón (cilindro central del radicchio) fue superior al requerido por la norma de calidad. Esto indica que estas plantas se debieron cosechar antes.

Cuadro 2. Porcentaje de radicchios recolectados según número de cosecha y variedad. Cartago, Costa Rica. 2005.

\begin{tabular}{lccc}
\hline \multirow{2}{*}{ Cosecha } & \multicolumn{2}{c}{ Variedad } & \\
\cline { 2 - 3 } & Verona & Leonardos & Total \\
\hline Primera & 49,1 & 67,0 & 58,0 \\
Segunda & 28,4 & 17,6 & 23,0 \\
Total & 77.5 & 84.6 & 81,0 \\
Perdidos & 22,5 & 15,4 & 19,0 \\
Total & 100,0 & 100,0 & 100,0 \\
\hline
\end{tabular}

La segunda cosecha fue ocho días después de la primera, a los 75 días del trasplante. Del total de radicchios de la variedad Leonardos obtenidos, excluyendo los no cabeceados (en la práctica éstos se habrían dejado en el campo), el 37,04\% no era adecuado para exportación por tener el corazón más grande que lo requerido. Esto demuestra que la variedad Leonardos, por su mayor crecimiento debe cosecharse más de una vez por semana, porque en menos de ocho días una cabeza que no está lista para cosecha puede superar el tamaño requerido.

Los resultados anteriores, comprueban lo citado por otros autores previamente (Rangarajan e Ingall 2001, Carrasco et al. 1998, Lazzarin et al. 2000, Carvajal y Krarup 1997) y muestran que es necesario evaluar la fecha de inicio de cosecha en las distintas épocas del año, puesto que lo usual para los agricultores en siembras de fin de invierno es iniciar la cosecha a los 70 días. De igual forma, los agricultores cosechan una vez por semana, sin embargo estos resultados indican que es necesario cosechar dos o más veces por semana, para evitar que las cabezas superen el tamaño requerido en el corazón.

Pese a que el Cuadro 2 muestra un porcentaje de pérdidas mayor para la variedad Verona que para la Leonardos, esta diferencia no es significativa, por lo que se considera aleatoria y por ende no generalizable. Con un 5\% de significancia ninguno de los efectos probados influyó en la cantidad de plantas perdidas (Cuadro 3).

Para estudiar la exportabilidad de los radicchios, suponiendo que se va a continuar con la práctica de cosechar únicamente en una determinada semana, se analizó la influencia de las distintas condiciones de cultivo en la exportabilidad obtenida a los 67 días de transplante. Para este análisis, los radicchios de la variedad Leonardos, que se consideraron pasados por el tamaño de su corazón, se tomaron como exportables, bajo el supuesto de que si se hubiese cosechado dos o más veces durante la semana de cosecha, habrían sido adecuados para la venta. Se encontraron dos efectos significativos (Cuadro 4): por cada ocho radicchios Leonardos exportables hay un radicchio Verona exportable y las plántulas grandes con cobertura plástica están en una desventaja de 0,33 a uno con respecto a las plántulas testigo sin acolchar.

El tamaño de las plántulas, por sí solo, no produjo diferencia en el porcentaje de plantas exportadas, posiblemente porque la uniformidad de las plántulas seleccionadas para el transplante indujo a un desarrollo uniforme de las plantas durante la etapa de producción, esto confirma con lo citado por Lazzarin et al. (2000). 
Cuadro 3. Intervalos de confianza y probabilidades asociadas a las razones de ventaja (odds) de cada efecto sobre el porcentaje de radicchios perdidos. Cartago, Costa Rica. 2005.

\begin{tabular}{lcccc}
\hline Efectos & $\begin{array}{c}\text { Razones } \\
\text { de } \\
\text { ventaja }\end{array}$ & $\begin{array}{c}\text { Probabili- } \\
\text { dad } \\
\text { asociada }\end{array}$ & $\begin{array}{c}\text { Intervalos de } \\
\text { confianza } \\
\mathbf{9 5 \%}\end{array}$ \\
\hline Variedad & 0,53 & 0,16 & 0,22 & 1,27 \\
Tamaño pequeño & 1,59 & 0,22 & 0,76 & 3,33 \\
Tamaño grande & 1,28 & 0,63 & 0,47 & 3,44 \\
Cobertura plástica & 0,63 & 0,15 & 0,33 & 1,19 \\
Tamaño pequeño x & 1,32 & 0,54 & 0,55 & 3,20 \\
Cobertura plástica & 1,12 & 0,84 & 0,38 & 3,30 \\
$\begin{array}{l}\text { Tamaño grande x } \\
\text { Cobertura plástica }\end{array}$ & & & & \\
$\begin{array}{l}\text { Variedad x Cober- } \\
\text { tura plástica }\end{array}$ & 0,74 & 0,49 & 0,31 & 1,74 \\
Variedad x Tamaño & 1,87 & 0,23 & 0,67 & 5,18 \\
pequeño & & & & \\
Variedad x Tamaño & 1,01 & 0,98 & 0,31 & 3,27 \\
grande & & & & \\
\hline
\end{tabular}

Cuadro 4. Intervalos de confianza y probabilidades asociadas a las razones de ventaja (odds) de cada efecto sobre la exportabilidad de radicchio en la primera cosecha. Cartago, Costa Rica. 2005.

\begin{tabular}{lcccc}
\hline Efectos & $\begin{array}{c}\text { Razones } \\
\text { de } \\
\text { ventaja }\end{array}$ & $\begin{array}{c}\text { Probabi- } \\
\text { lidad } \\
\text { asociada }\end{array}$ & $\begin{array}{c}\text { Intervalos de } \\
\text { confianza al } \\
\mathbf{9 5 \%}\end{array}$ \\
\hline Variedad & 8,13 & 0,00 & 3,33 & 19,90 \\
Tamaño pequeño & 1,09 & 0,87 & 0,41 & 2,87 \\
Tamaño grande & 2,27 & 0,09 & 0,89 & 5,78 \\
Cobertura plástica & 2,21 & 0,09 & 0,88 & 5,55 \\
Tamaño pequeño * & & & & \\
Cobertura plástica & 0,43 & 0,10 & 0,16 & 1,16 \\
Tamaño grande * & & & & \\
Cobertura plástica & 0,33 & 0,04 & 0,11 & 0,95 \\
$\begin{array}{l}\text { Variedad *Cober- } \\
\text { tura plástica }\end{array}$ & 0,86 & 0,73 & 0,36 & 2,03 \\
Variedad * Tama- & & & & \\
ño pequeño & 0,64 & 0,39 & 0,23 & 1,76 \\
Variedad * Tama- & & & \multicolumn{3}{c}{0,25} & 2,13 \\
\hline ño grande & 0,73 & 0,56 & 0,25 \\
\hline
\end{tabular}

Para el análisis de la exportabilidad total (ambas cosechas), lo único que afectó este factor fue la variedad (Cuadro 5). Por cada nueve radicchios exportables de la variedad Leonardos hay uno de la variedad Verona. Dado que el tamaño del almácigo no hace diferencia en la exportabilidad total, ni en el porcentaje exportado si se cosechara en una única semana dada, se podría usar almácigo más pequeño o más grande que el usual, siempre y cuando se asegure una alta uniformidad del almácigo, lo cual representa una ventaja de manejo para el agricultor.

A pesar de existir la teoría de que la cobertura con plástico aumenta la producción, en este caso no produjo beneficio alguno en contraste con lo encontrado por Rangarajan e Ingall (2001), en investigaciones realizadas en el estado de New York en los Estados Unidos, bajo condiciones de clima muy diferentes a las prevalentes en este experimento. Este resultado podría estar dado por la época en que se realizó el experimento (periodo lluvioso) en donde los altos niveles de humedad en el suelo y la baja radiación pudieron reducir el efecto beneficioso que se cita para el uso del plástico.

Cuadro 5. Intervalos de confianza y probabilidades asociadas a las razones de ventaja (odds) de cada efecto sobre la exportabilidad total del radicchio. Cartago, Costa Rica. 2005.

\begin{tabular}{lcccc}
\hline Efectos & $\begin{array}{c}\text { Razones } \\
\text { de } \\
\text { ventaja }\end{array}$ & $\begin{array}{c}\text { Probabi- } \\
\text { lidad } \\
\text { asociada }\end{array}$ & $\begin{array}{c}\text { Intervalos de } \\
\text { confianza al } \\
\mathbf{9 5 \%}\end{array}$ \\
\hline Variedad & 9,10 & 0,00 & 3,63 & 22,81 \\
Tamaño pequeña & 1,30 & 0,59 & 0,50 & 3,41 \\
Tamaño grande & 1,82 & 0,18 & 0,75 & 4,43 \\
Cobertura plástica & 2,26 & 0,08 & 0,90 & 5,67 \\
$\begin{array}{l}\text { Tamaño pequeño * } \\
\text { Cobertura plástica }\end{array}$ & 0,44 & 0,15 & 0,14 & 1,36 \\
$\begin{array}{l}\text { Tamaño grande * } \\
\text { Cobertura plástica }\end{array}$ & 0,50 & 0,19 & 0,18 & 1,40 \\
$\begin{array}{l}\text { Variedad * Cober- } \\
\text { tura plástica }\end{array}$ & 0,95 & 0,92 & 0,39 & 2,33 \\
$\begin{array}{l}\text { Variedad * Tamaño } \\
\text { pequeño }\end{array}$ & 0,57 & 0,34 & 0,19 & 1,77 \\
$\begin{array}{l}\text { Variedad * Tamaño } \\
\text { grande }\end{array}$ & 0,78 & 0,65 & \multicolumn{3}{|c}{0,28} & 2,21 \\
\hline
\end{tabular}


Sería necesario conducir un nuevo experimento en el periodo seco para verificar si el cambio de estación mejora la respuesta del plástico.

En el Cuadro 6 se observa que la mayor parte de los rechazos de radicchios de la variedad Leonardos, se dieron por falta de cabeceo $(76,9 \%)$, o sea que en este caso el acomodo de las hojas no lograron compactarse y formar una "cabeza"; las otras causas de rechazo fueron poco importantes en términos relativos. En Verona la causa principal fue cabeceado suave $(52,9 \%)$, o sea que sí se formó la "cabeza", pero ésta no alcanzó el grado de compactación suficiente para cumplir con la norma de calidad exigida por el mercado, el no cabeceo representó el $24,8 \%$, leve color morado $(17,8 \%)$ y por tamaño pequeño los rechazos fueron muy pocos.

Cuadro 6. Radicchios rechazados según razón de rechazo y variedad. Cartago, Costa Rica. 2005.

\begin{tabular}{lcrrr}
\hline \multirow{2}{*}{$\begin{array}{c}\text { Razones de } \\
\text { rechazo }\end{array}$} & \multicolumn{4}{c}{ Variedad } \\
\cline { 2 - 5 } & \multicolumn{2}{c}{ Leonardos } & \multicolumn{2}{c}{ Verona } \\
\cline { 2 - 5 } & \multicolumn{1}{c}{$\mathbf{n}$} & $\boldsymbol{1}$ & \multicolumn{1}{c}{ n } & $\%$ \\
\hline Color & 3 & 7,7 & 28 & 17,8 \\
Pequeño & 1 & 2,6 & 7 & 4,5 \\
Cabeceado suave & 5 & 12,8 & 83 & 52,9 \\
No cabeceado & 30 & 76,9 & 39 & 24,8 \\
Total & 39 & 100,0 & 157 & 100,0 \\
\hline
\end{tabular}

De los radicchios de la variedad Leonardos cosechados (exceptuando los no cabeceados) un 13,08\% fue rechazado por el personal de empaque porque se consideraron pasados (con mayor desarrollo que el solicitado por el mercado), cuando en realidad no lo estaban y por el contrario, un 28,69\% fue declarado exportable, cuando el tamaño del "corazón" (cilindro central del radicchio) era muy alto para ser exportado.

En cuanto al criterio del color adecuado para exportar, 138 radicchios declarados exportables, tenían grados de color entre dos y tres, mientras que 27 radicchios que tenían estos mismos grados, fueron rechazados por falta de color morado.
De los radicchios de la variedad Verona cosechados, exceptuando los no cabeceados, un 39,15\% fue rechazado por el empacador por cabeceado suave, este resultado indica que existe una divergencia entre lo que el cosechador y el empacador perciben al tacto como cabeza firme, ya que si sus criterios estuvieran unificados, el rechazo por cabeceado suave debería ser muy bajo o nulo.

Los resultados anteriores confirman que existe la necesidad de capacitar a los productores y a sus empleados en lo que se refiere a unificación de criterios sobre el cumplimiento de las normas de calidad, con el fin de evitar pérdidas de producto y mejorar la calidad de exportación.

\section{CONCLUSIONES}

La variedad Leonardos tuvo una mayor exportabilidad que la variedad Verona. La baja exportabilidad de esta última puede deberse a que la semilla que se utiliza no es F1, por lo cual su desarrollo es muy poco uniforme, así como también por divergencias entre el cosechador y el empacador del producto en lo percibido al tacto como cabeza firme.

Es posible uniformar la fecha de cosecha con solo asegurar la uniformidad del tamaño de las plántulas al transplante. El tamaño de plántulas no ejerció influencia sobre la exportabilidad en términos de uniformidad del desarrollo de la planta para llegar al punto de cosecha, posiblemente porque la uniformidad en el desarrollo de las plántulas seleccionadas para el transplante fue mayor que la usada por el agricultor.

Como el tamaño de plántulas no produjo diferencias, se podría usar almácigos más pequeños o más grandes que el usual, siempre y cuando se garantice la uniformidad del tamaño de las plántulas.

Al no ejercer la cobertura con plástico un efecto significativo sobre la productividad, no es adecuada su práctica, ya que aumentarían los costos de producción.

Existieron problemas con la unificación del criterio de exportabilidad entre los cosechadores y los empacadores, esto produce pérdidas de producto que no puede ser exportado porque fue cosechado en forma temprana o tardía. 
El tiempo de inicio de cosecha utilizado por los agricultores no es el más apto. El estudio indica que la cosecha se debe iniciar antes de los 67 días del trasplante, sin embargo se debe seguir prestando mucha atención a las fechas de inicio de cosecha en las distintas épocas del año. Además es recomendable cosechar dos o más veces por semana.

Las recomendaciones derivadas de este estudio fueron implementadas en la misma finca dos meses después en dos hectáreas de cultivo. El nivel del producto exportado del 74,6\% obtenido para la variedad Leonardos en el experimento, se elevó a un $92 \%$ en ese cultivo comercial.

\section{LITERATURA CITADA}

Carrasco, G; Carmona, C; Sandoval, C; Urrestarazu, M. 1998. Plant density on yield of red chicory heads-radicchio rosso (Cichorium intybus var. foliosum) grow in south central Chile. Acta Horticulturae (467): 269-275.

Carvajal, M; Krarup, HA. 1997. Performance of several cultivars of raddichio Ander the conditions of Valdivia. Agro Sur 25(1): 57-61.

Lazzarin, R; Bellia, L; Masiero, D. 2000. Nuove opportunita per il trapianto del radicchio. Informatore Agrario 56(26): 76-79.
Pimpini, F, Lazzarin, R, Chillemi, G. 2002. Aspetti generali. In: I. De Maria, I. Lavezzo, A. Tadiotto eds. Il Radicchio Variegato di Castelfranco. Veneto agricoltura, Veneto, Italia. p. 15-43.

Ramponi, E. 2000. Le tecniche di produzione e difesa del radicchio rosso tardivo di Treviso. Revista L'Informatore Agrario 56: 43-47.

Rangarajan, A; Ingall, B. 2001. Mulch color affects radicchio quality and yield. HortScience 36(7): 1240-1243.

Reich, HV; Coffey, LD; Summerlin, J. 2000. Evaluation of radicchio, plant science farm, Knoxville Experiment". (en línea). Consultado abril 2005. Disponible en: http://bioengr.ag.utk.edu/Extension/ExtProg/Vegetable/year/VegInit.

Reich, HV; Coffey, LD. 2001. Studies on radicchio. University of Tennessee Experiment Station. (en línea). Consultado abril 2005. Disponible en: http:// bioengr.ag.utk.edu/Extension/ExtProg/Vegetable/ year/VegInit.

Solutions Associates, 2006. Oportunidades para Chile en la exportación de vegetales de alto valor en el mercado de EEUU. Fundación Chile, Santiago, Chile. 77 p.

Suhonen, I. 1991. Growth bolting and yield quality of "radicchio rosso". Scientia Horticulturae 46 (1-2): 25-31. 